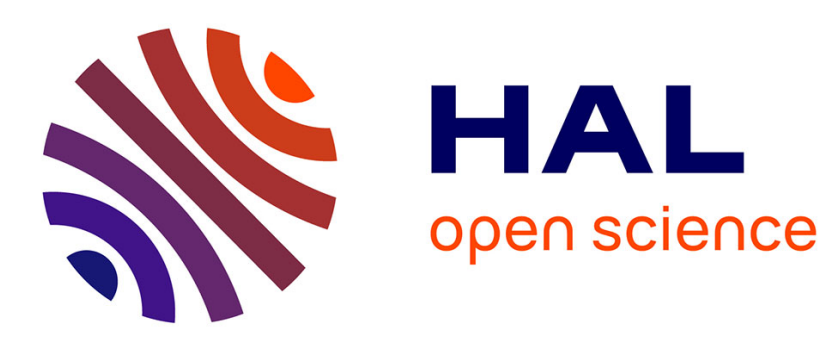

\title{
Full nuclear field theory treatment of two-particle-one-hole-excitations
}

\author{
B. Silvestre-Brac, R.J. Liotta
}

\section{To cite this version:}

B. Silvestre-Brac, R.J. Liotta. Full nuclear field theory treatment of two-particle-one-hole-excitations. Journal de Physique, 1981, 42 (6), pp.799-811. 10.1051/jphys:01981004206079900 . jpa-00209066

\section{HAL Id: jpa-00209066 https://hal.science/jpa-00209066}

Submitted on 1 Jan 1981

HAL is a multi-disciplinary open access archive for the deposit and dissemination of scientific research documents, whether they are published or not. The documents may come from teaching and research institutions in France or abroad, or from public or private research centers.
L'archive ouverte pluridisciplinaire HAL, est destinée au dépôt et à la diffusion de documents scientifiques de niveau recherche, publiés ou non, émanant des établissements d'enseignement et de recherche français ou étrangers, des laboratoires publics ou privés. 


\title{
Full nuclear field theory treatment of two-particle-one-hole-excitations (*)
}

\author{
B. Silvestre-Brac \\ Institut des Sciences Nucléaires, 53, avenue des Martyrs, 38026 Grenoble, France \\ and R. J. Liotta $\left({ }^{* *}\right)$ \\ Laboratoire de Physique Théorique (***), Université de Bordeaux-I, \\ chemin du Solarium, 33170 Gradignan, France \\ (Reçu le 10 juin 1980, révisé le 6 fẹvrier 1981, accepté le 18 février 1981)
}

\begin{abstract}
Résumé. - Pour décrire les excitations à 2 particules-1 trou, nous faisons la somme de tous les diagrammes de perturbation de la théorie du champ nucléaire en incluant seulement les sommets Tamm-Dancoff. Il est montré que la théorie donne les mêmes résultats que ceux fournis par la méthode du modèle en couches, mais seulement si tous les états de base possibles sont introduits dans le formalisme. L'applicabilité de la théorie est discutée dans un modèle simple.
\end{abstract}

\begin{abstract}
The nuclear field theory series is summed up to all orders of perturbation theory including only Tamm-Dancoff vertices for the case of two-particle-one-hole-excitations. It is found that the theory gives the same results as those provided by the shell-model method, but only if all possible basis states are included in the formalism. Applicability of the theory is discussed in a simple model.
\end{abstract}

1. Introduction. - For quantum systems - like nuclei - having many degrees of freedom, the corresponding total Hilbert space is tremendously large. The usual way to tackle the problem is to get an approximate wavefunction as a superposition of independent particle wavefunctions. The number of single particle states is reduced to a reasonable limit and the realistic two-body interaction is replaced in a coherent way by an effective two-body interaction. The number and the nature of the single-particle orbits where the nucleons are allowed to move and the number of valence particles define the configuration space. The full shell-model consists in calculating the matrix of the effective interaction in all the possible vectors of the configuration space and diagonalizing it. In principle a comparison between the full shell-model results and the experimental ones provides indications on how good is the effective interaction. However the above configuration space including many-particle-many-hole-excitations is still too large for practical applications and one needs

$\left({ }^{*}\right)$ Work supported by NORDITA.

(**) Permanent address : Research Institute for Physics, S-10405 Stockholm 50, Sweden.

$\left({ }^{* * *}\right)$ Equipe de Recherche Associée au C.N.R.S. further approximations. Generally it is supposed that the low-lying states are rather well described with few-particle-few-hole-excitations. Even with this simplified space (here after called shell-model space) the shell-model calculations may be very difficult to perform and other approaches are necessary. The use of correlated states to describe nuclear spectra has lately been well accepted [1-5]. In general, these models using correlated states try to reproduce shell-model results. Thus, if a given calculation provided by a certain method can reproduce the corresponding shell-model results, one feels confident in making approximation within that method. Usually, the most important approximation that one has in mind is to drastically truncate the dimension of the shell-model space. One then hopes that the physical solutions are included in the relatively small subspace which is left after the truncation. This is one important reason why so many methods and models that include correlated states in the basis have been proposed.

Among these models, the nuclear field theory [5] (NFT) has been applied to analyse a wide range of physical processes like nuclear spectroscopy [5], alpha decay [6] and nuclear reactions [7]. The nuclear field theory is a method which is based on a dia- 
grammatic perturbation series. The number and the complexity of the resulting diagrams depend on the nature of the system actually studied. In most of the applications done so far within the framework of the NFT, only collective bosons have been considered. Generally the collective bosons are associated with single-particle shells that are highly degenerated. For cases like these, the application of NFT to first order of perturbation ought to be justified. One expects that higher orders would give a contribution smaller than $1 /\left(2 \Omega_{\text {eff }}\right)$, where $\Omega_{\text {eff }}$ is the effective degeneracy of the single-particle shells where the nucleons are allowed to move [5-6]. However, recently the importance of non-collective bosons have also been attested for describing some excited states $[8,14]$.

In any case the restriction to lowest order diagrams is very questionable. In case of disagreement between theory and experiment it is difficult to say, a priori, whether the discrepancy comes from some physical deficiencies already present in the shell-model (bad interaction, higher order excitations) or from the importance of the neglected diagrams. One way to answer this question is to perform the whole summation of the diagrammatic series. This has been done only for some very simple models (one or two levels) with special type of interaction (pairing interaction). Recently however [8] we gave a method allowing to treat exactly the NFT series for the case of a realistic system (arbitrary number of shells and interaction) containing three particles outside a closed shell core. The only requirement was the restriction to diagrams including only TDA vertices. This condition is completely equivalent to neglect particle-hole-excitations (even as virtual excitations). The main results of reference [8] are that if the whole diagrammatic series is summed up i) the spurious states coming from the violation of the Pauli principle are correctly eliminated on grounds of energy and norm considerations; ii) the nuclear field theory reproduces the exact shell-model results. The three particle system analysed in reference [8] was the simplest that one could find not only because the number of excitation was small but also because only pairing (particle-particle) bosons were included. As a consequence only the particle-particle interactions could be tested.

The generalization of the procedure given in [8] to more complex situations is not a straightforward task. One aim of this paper is to show how to perform such a generalization for the case of two-particle-onehole-excitations. In that case not only pairing bosons are present but also surface bosons (particle-hole) allowing thus the simultaneous test of the particleparticle and particle-hole interactions. The corresponding formalism is developed in section 2 . The method is then applied to a two level model where an analytical NFT calculation and an exact shellmodel treatment can easily be done. A more elaborated model is used to discuss the influence of the collectivity of the bosons and the effect of a trunca- tion in the basis. Another aim of the paper is to see, through the illustrative examples of sections 3 and 4 , if the conclusions of reference [8] are still valid in this more complicated case and to test the applicability of the method for removing the spurious states. Discussions and conclusions are presented in the last section.

2. Formalism. - The NFT diagrammatic series is rather peculiar in that the basis elements are essentially different to the intermediate state elements. In the basis, no single-particle configuration which can be a combination of boson states is allowed [9]. For the two-particle-one-hole-excitations that we study here, this rule means that the basis consists of the elements shown in figure 1 . The elements $1 a$ and $1 b$ have been included already in the first calculation done within the framework of the NFT ten years ago [10]. However, diagrams $1(c-d)$ have never been used in NFT calculations. This is intuitively justified by saying that already the diagram $1 a$ is more than enough to describe $2 \mathrm{p}-1 \mathrm{~h}$-excitations [11]. Indeed, if one thinks in the corresponding singleparticle description of figure $1 a$ one would think that diagram $1 a$ provides an overcomplete basis since one neglects the Pauli principle between the free particle and the particle that constitutes the boson. Although the Pauli principle is corrected within the NFT perturbation series $[5,8]$ the fact that the basis provides more states than those allowed by the Pauli principle remains. However, an important feature of the NFT bosons is that they are independent of the constituents degrees of freedom. These bosons are real bosons (in contrast to the «bosons" in any of the methods in references [2-4]) so that the NFT basis elements are orthonormal to each other and, therefore, the NFT basis is complete. Yet, since an exact calculation within the NFT would give a number of spurious states one generally uses a loose language and speaks of the "overcomplete " NFT basis meaning that this basis spans a space which is larger than the physical space. Following this bad tradition we will use in this paper the same language and call "overcomplete" the complete NFT basis. As seen in figure 1, in our case the basis is heavily overcomplete. In the next sections, we will analyse in more detail problems which arise because of this NFT feature.

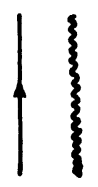

(a)

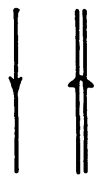

(b)

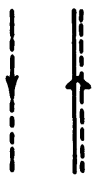

(c)

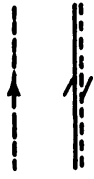

(d)
Fig. 1. - NFT basis elements. Full (dashed) lines represent neutrons (protons) states or vice versa.

Another reason why diagrams $1 c-d$ have so far not been considered in the NFT basis is that they do not 
include collective bosons. Thus, one expects that their influence is negligible. However, one sometimes finds that non-collective bosons may also be important in describing physical states $[8,14]$.

To derive the NFT equations to all orders of perturbation theory including all the basis elements shown in figure 1 , we consider only TDA vertices. We should thus obtain the same solutions than those provided by the shell-model for the $2 \mathrm{p}$ - $1 \mathrm{~h}$-excitations.

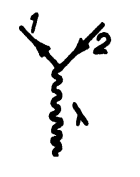

(a)

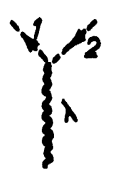

(d)

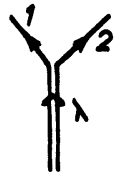

(b)
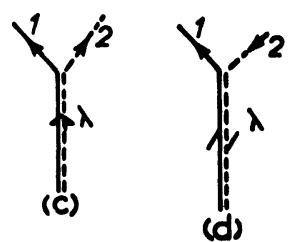

Fig. 2. - Particle-boson coupling vertices that enter in our calculations.

The TDA vertices are depicted in figure 2. In figures $2 a$ and $2 a^{\prime}$ the wavy line means, as usual, particle-hole (proton-proton and neutron-neutron) boson while the double line of figure $2 b$ represents the two-particle (proton-proton or neutron-neutron) boson, also as usual [5]. In figures $2 c$ and $2 d$ dotted lines denote proton (neutron) states if full lines denote neutron (proton) states [6]. It is rather straightforward to see that there is not a formal difference between diagrams $2 a$ and $2 d$ neither between $2 b$ and $2 c$. For instance, vertices $2 a$ and $2 d$ have the value

$$
\left\langle 0\left|\left(c_{1}^{\dagger} b_{2}^{\dagger}\right)_{\lambda \mu}^{\dagger} H_{\text {p.v. }} \Gamma_{n}^{\dagger}(0 \lambda \mu)\right| 0\right\rangle=\Lambda_{n}(12,0 \lambda)
$$

where

$$
b_{j m}^{\dagger}=(-1)^{j-m} c_{j-m}
$$

and $c_{i}^{\dagger}$ is the creation operator of a nucleon in the state with quantum numbers $i$ (which includes the component $t_{z}$ of iso-spin). The rest of the quantities in equation (1) are the particle-boson coupling hamiltonian $H_{\text {p.v. }}$, the boson creation operator $\Gamma^{\dagger}$ and the corresponding coupling constant $\Lambda$ (see Ref. [12]). In the same fashion, one can keep track of all contributions with dotted lines in any diagram. From now on we will consider, from a formal viewpoint, only full line diagrams. All the others come naturally through the iso-spin components in the quantum numbers that identify each line. We apply in this paper the diagrammatic Brillouin-Wigner (B-W) perturbation theory [5]. Then, all the corresponding diagrams with TDA vertices shown in figure 3 can be studied analysing only those diagrams in figures $3 a-d$. Below we show how to sum-up the whole series of diagrams of figure 3 . These diagrams are built according to the B-W perturbation method. If one applies, for instance, the Rayleigh-Schrödinger perturbation method $[5,6]$ the number of diagrams becomes so large that we found no way of performing the whole summation. Even in the B-W case the number of diagrams to be considered is very large. One finds that in figure $3 a$ there is $N_{1}=1$ diagram in first order, $N_{2}=2$ in second order and, in general, there are $N_{n}=N_{n-1}+N_{n-2}$ diagrams of order $n$. The number $N_{n}$, which is the $n$th term of the Fibonacci sequence, has the value $\left(\Phi_{(+)}^{n+1}-\Phi_{(-)}^{n+1}\right) / \sqrt{5}$, where

$$
\Phi_{( \pm)}=(1 \pm \sqrt{5}) / 2 \text {. }
$$

Therefore the number of diagrams increases exponentially with the order of perturbation $n$ (as compared with $N_{n}=1$ for the three-particle system [8]). To sum-up the series of diagrams of figure 3 one can follow two different approaches, as shown in figures 4 and 7.

In figure 4 we define the propagators $A_{\sigma(\pi)}$ and $B_{\sigma(\pi)}$ such that they describe the propagation of the $2 \mathrm{p}-1 \mathrm{~h}$ excitation from the intermediate state shown in the figure to the final state $\sigma(\pi)$, where $\sigma(\pi)$ labels the basis states as

$$
\begin{aligned}
|\sigma\rangle & \equiv\left[c_{k}^{\dagger} \times \Gamma_{n}^{\dagger}\left(\begin{array}{ll}
0 & \lambda
\end{array}\right)\right]_{M}^{I}|0\rangle \\
|\pi\rangle & \equiv\left[b_{i}^{\dagger} \times \Gamma_{n}^{\dagger}(2 \lambda)\right]_{M}^{I}|0\rangle .
\end{aligned}
$$

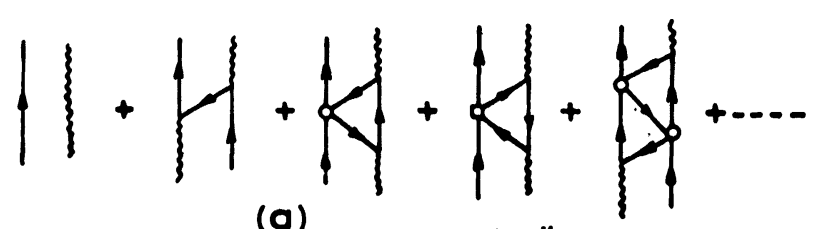

(a)

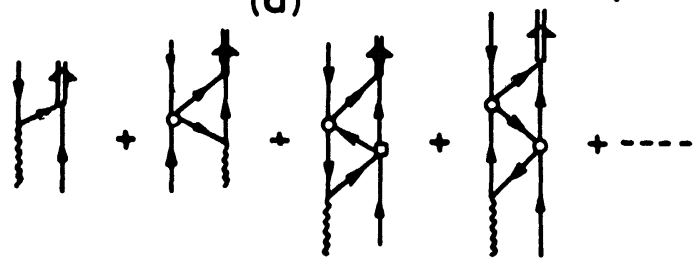

(b)
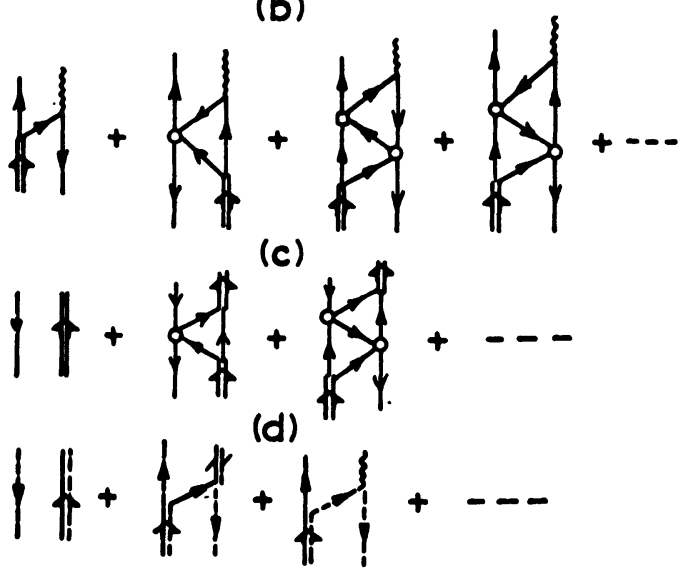

(e)

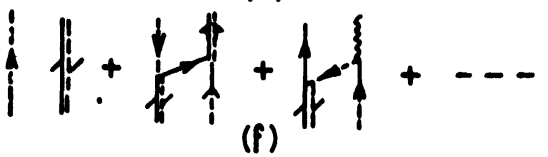

Fig. 3. - Graphical series provided by the NFT perturbation method. The circles in the bare-interaction vertices indicate particlehole interactions while the squares indicate particle-particle interactions. 
In these equations, as well as throughout this paper, $k(i)$ labels single-particle (single-hole)-excitations. The operator $\Gamma_{n}^{\dagger}(0 \lambda)\left(\Gamma_{n}^{\dagger}(2 \lambda)\right)$ creates the $n$th particle-hole (particle-particle) boson with angular momentum $\lambda$ (the number $n$ also includes $t_{z}$ ). The total angular momentum of the $2 \mathrm{p}-1 \mathrm{~h}$ system is $I$. We will use the letter $\rho$ to denote either state of the type $\sigma$ or $\pi$. Latin letters label single-particle states but to simplify the writing of our formulas we also use the same labels to indicate only the total angular momenta (i.e., in Eq. (6) $(-1)^{k-k_{1}} \equiv(-1)^{j_{k}-j_{k_{1}}}$.
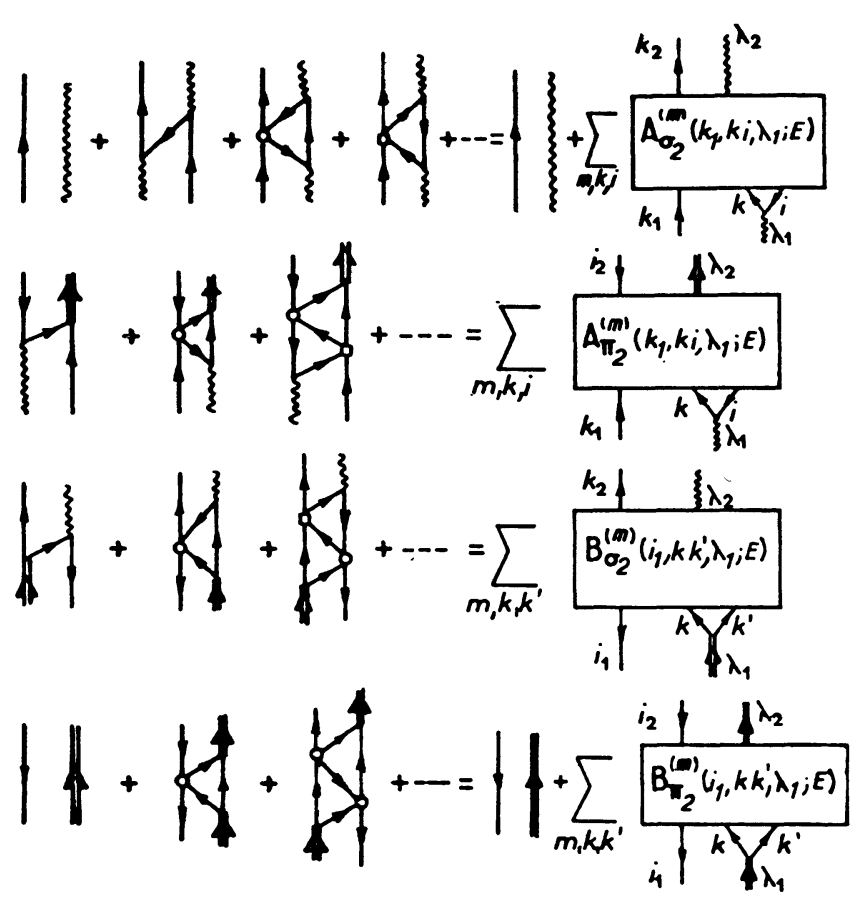

Fig. 4. - Graphical representation of the propagators $A_{\rho}$ and $B_{\rho}$.
From figure 4 one can write, for instance,

$$
\begin{aligned}
& W_{\sigma \sigma^{\prime}}(E)=\left(\varepsilon_{k}+\omega_{n}(0 \lambda)\right) \delta\left(\sigma, \sigma^{\prime}\right)+\sum_{m} W_{\sigma \sigma^{\prime}}^{(m)}(E) \\
& W_{\sigma \sigma^{\prime}}^{(m)}(E)=\sum_{k_{1} i_{1}} A_{\sigma}^{(m)}\left(k^{\prime}, k_{1} i_{1}, \lambda^{\prime} ; E\right) \Lambda_{n^{\prime}}\left(k_{1} i_{1}, 0 \lambda^{\prime}\right)
\end{aligned}
$$

where $m$ denotes the order of perturbation, and similar expressions for the other matrix elements of the NFT effective interaction $W$.

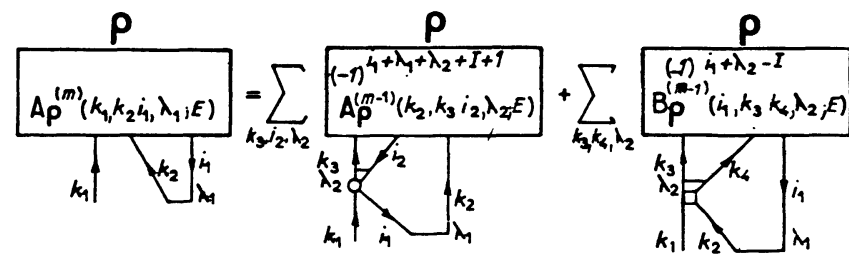

(a)

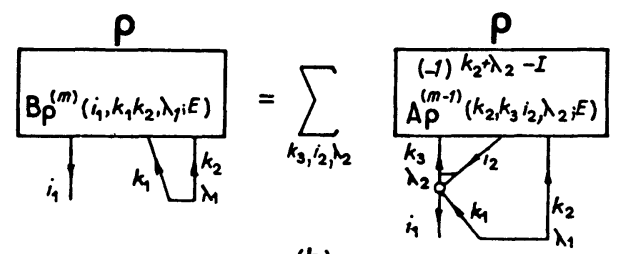

(b)

Fig. 5. - Recurrence relation for the propagators $A_{\rho}(\mathrm{a})$ and $B_{\rho}(\mathrm{b})$. The horizontal bar joining two lines in the propagators $A$ and $B$ means that these two lines represent states coupled to the angular momentum given by the bar.

One can evaluate the propagators $A$ and $B$ making use of the diagrams in figure 5 to obtain (see appendix)

$$
\left(\begin{array}{cc}
I-K_{a a}(E) & -K_{a b}(E) \\
-K_{b a}(E) & I
\end{array}\right)\left(\begin{array}{c}
A_{\sigma(\pi)} \\
B_{\sigma(\pi)}
\end{array}\right)=\left(\begin{array}{c}
A_{\sigma(\pi)}^{(1)} \\
B_{\sigma(\pi)}^{(1)}
\end{array}\right)
$$

where the first order contributions $A^{(1)}$ and $B^{(1)}$ are (Fig. 6)

$$
\begin{aligned}
& A_{\sigma}^{(1)}\left(k_{1}, k_{2} i_{1}, \lambda_{1} ; E\right)=\delta\left(k k_{2}\right)(-1)^{k-k_{1}+\lambda-\lambda_{1}} \hat{\lambda} \hat{\lambda}_{1}\left\{\begin{array}{ccc}
k_{2} & i_{1} & \lambda_{1} \\
k_{1} & I & \lambda
\end{array}\right\} \Lambda_{n}^{*}\left(k_{1} i_{1}, 0 \lambda\right)\left[E-\left(\varepsilon_{k_{1}}+\varepsilon_{k_{2}}-\varepsilon_{i_{1}}\right)\right]^{-1} \\
& B_{\sigma}^{(1)}\left(i_{1}, k_{1} k_{2}, \lambda_{1} ; E\right)=\delta\left(k k_{2}\right) \hat{\lambda} \hat{\lambda}_{1}\left\{\begin{array}{lll}
k_{2} & k_{1} & \lambda_{1} \\
i_{1} & I & \lambda
\end{array}\right\} \Lambda_{n}^{*}\left(k_{1} i_{1}, 0 \lambda\right)\left[E-\left(\varepsilon_{k_{1}}+\varepsilon_{k_{2}}-\varepsilon_{i_{1}}\right)\right]^{-1} \\
& A_{\pi}^{(1)}\left(k_{1}, k_{2} i_{1}, \lambda_{1} ; E\right)=\delta\left(i, i_{1}\right) \hat{\lambda} \hat{\lambda}_{1}\left\{\begin{array}{lll}
i_{1} & k_{2} & \lambda_{1} \\
k_{1} & I & \lambda
\end{array}\right\} \Lambda_{n}^{*}\left(k_{2} k_{1}, 2 \lambda\right) N\left(k_{1} k_{2}\right)\left[E-\left(\varepsilon_{k_{1}}+\varepsilon_{k_{2}}-\varepsilon_{i_{1}}\right)\right]^{-1} \\
& B_{\pi}^{(1)}\left(i_{1}, k_{1} k_{2}, \lambda_{1} ; E\right)=0
\end{aligned}
$$

where $N\left(k_{1} k_{2}\right)=\left(1+\delta\left(k_{1}, k_{2}\right)\right)^{1 / 2}$. As seen in figure $6 d, B_{\pi}^{(1)}$ vanishes because the corresponding diagram would include bubbles, which are forbidden by one of the NFT rules [9].

Using the diagrams in figure 5 one can readily calculate the matrices $K_{a a} K_{a b}$ and $K_{b a}$, as shown in the appendix. 

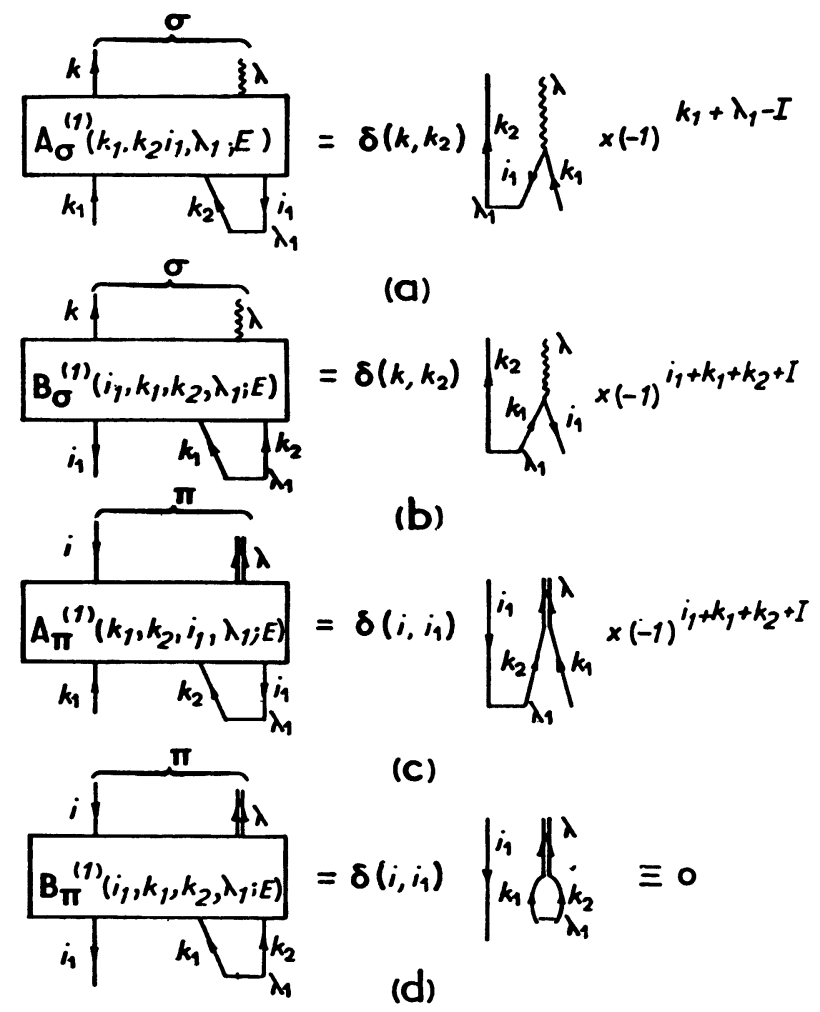

Fig. 6. - First order representation of the propagators $A_{\rho}$ and $B_{\rho}$.

The interaction matrix elements $W$ can also be evaluated through the propagators $C$ and $D$ of figure 7 . One gets, i.e.

$$
\begin{aligned}
W_{\sigma \sigma^{\prime}}(E)= & \left(\varepsilon_{k}+\omega_{n}(0 \lambda)\right) \delta\left(\sigma, \sigma^{\prime}\right)+\sum_{k_{1} i_{1} \lambda_{1}}(-1)^{k_{1}-k+\lambda_{1}-\lambda} \hat{\lambda} \hat{\lambda}_{1}\left\{\begin{array}{lll}
k & i_{1} & \lambda_{1} \\
k_{1} & I & \lambda
\end{array}\right\} \times \\
& \times \Lambda_{n}^{*}\left(k_{1} i_{1}, 0 \lambda\right) C_{\sigma^{\prime}}\left(k_{1}, k i_{1}, \lambda_{1} ; E\right)+\sum_{k_{1} i_{1} \lambda_{1}} \hat{\lambda} \hat{\lambda}_{1}\left\{\begin{array}{lll}
k & k_{1} & \lambda_{1} \\
i_{1} & I & \lambda
\end{array}\right\} \Lambda_{n}^{*}\left(k_{1} i_{1} ; 0 \lambda\right) D_{\sigma^{\prime}}\left(i_{1}, k_{1} k, \lambda_{1} ; E\right) .
\end{aligned}
$$

As before one can write the matrix equation for $C$ and $D$ as

$$
\left(\begin{array}{cc}
I-K_{c c}(E) & -K_{c d}(E) \\
-K_{d c}(E) & I
\end{array}\right)\left(\begin{array}{c}
C_{\sigma(\pi)} \\
D_{\sigma(\pi)}
\end{array}\right)=\left(\begin{array}{c}
C_{\sigma(\pi)}^{(1)} \\
D_{\sigma(\pi)}^{(1)}
\end{array}\right)
$$

the matrices $K_{c c} K_{c d}$ and $K_{d c}$ as well as $C^{(1)}$ and $D^{(1)}$ are given in the appendix.

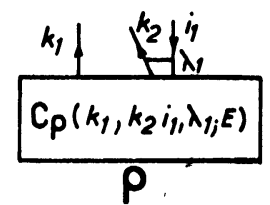

(a)

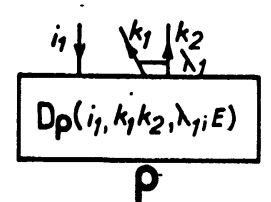

(b)

Fig. 7. - Graphical representation of the propagators $C_{\rho}$ and $D_{\rho}$.

From (A.5) and (A.6) one can show that the following symmetry relation holds

$$
D_{\rho}\left(i_{1}, k_{1} k_{2}, \lambda_{1} ; E\right)=(-1)^{k_{1}+k_{2}+\lambda_{1}+1} D_{\rho}\left(i_{1}, k_{2} k_{1}, \lambda_{1} ; E\right) .
$$

There is not a similar relation for the matrix $B$. The property (9) makes it more convenient to calculate the matrix elements of the interaction $W$ through $C$ and $D$ instead of $A$ and $B$. Yet, these matrices are not independent 
to each other. After some algebra one finds

$$
\begin{gathered}
A_{\rho}\left(k_{1}, k_{2} i_{1}, \lambda_{1} ; E\right)=\sum_{\lambda_{2}} \hat{\lambda}_{1} \hat{\lambda}_{2}\left[\left\{\begin{array}{lll}
k_{1} & k_{2} & \lambda_{2} \\
i_{1} & I & \lambda_{1}
\end{array}\right\} D_{\rho}^{*}\left(i_{1}, k_{2} k_{1}, \lambda_{2} ; E\right)+\right. \\
\left.+(-1)^{k_{1}-k_{2}+\lambda_{1}-\lambda_{2}}\left\{\begin{array}{lll}
k_{1} & i_{1} & \lambda_{2} \\
k_{2} & I & \lambda_{1}
\end{array}\right\} C_{\rho}^{*}\left(k_{2}, k_{1} i_{1}, \lambda_{2} ; E\right)\right] . \\
B_{\rho}\left(i_{1}, k_{1} k_{2}, \lambda_{1} ; E\right)=P\left(k_{1} k_{2}, \lambda_{1}\right) \sum_{\lambda_{2}} \hat{\lambda}_{1} \hat{\lambda}_{2}\left\{\begin{array}{lll}
i_{1} & k_{1} & \lambda_{2} \\
k_{2} & I & \lambda_{1}
\end{array}\right\} C_{\rho}^{*}\left(k_{2}, k_{1} i_{1}, \lambda_{2} ; E\right)
\end{gathered}
$$

the quantity $P=0$ if $k_{1}=k_{2}$ and $\lambda_{1}$ is odd, otherwise $P=1$.

When all matrices have been evaluated one can proceed to calculate the energies and wavefunctions as

$$
W(E) \Psi_{n}\left(E_{n}\right)=E_{n} \Psi_{n}\left(E_{n}\right)
$$

which is actually a self-consistent problem [8] since one has to find the value $E$ in $W(E)$ such that $E=E_{n}$. The wavefunctions are then written as

$$
\left|\Psi_{n}\right\rangle=\sum_{i} \xi_{i}^{(n)}\left|\rho_{i}\right\rangle
$$

The orthonormality condition for the wavefunction (12) is

$$
\left\langle\Psi_{m} \mid \Psi_{n}\right\rangle=\delta(m, n)=\sum_{i, j}\left(\delta(i, j)+N_{i j}^{m n}\right) \xi_{i}^{(m) *} \xi_{j}^{(n)}
$$

where the matrix $N$ reflects the effect of the intermediate states upon the basis states. It can be calculated graphically, as shown in figure 8 , to get

$$
N_{i j}^{m n}=\sum_{i_{1} k_{1} k_{2} \lambda_{1}}\left(B_{\rho_{i}}\left(i_{1}, k_{1} k_{2}, \lambda_{1} ; E_{m}\right) D_{\rho_{j}}\left(i_{1}, k_{1} k_{2}, \lambda_{1} ; E_{n}\right)+A_{\rho_{i}}\left(k_{1}, k_{2} i_{1}, \lambda_{1} ; E_{m}\right) C_{\rho_{j}}\left(k_{1}, k_{2} i_{1}, \lambda_{1} ; E_{n}\right)\right) .
$$

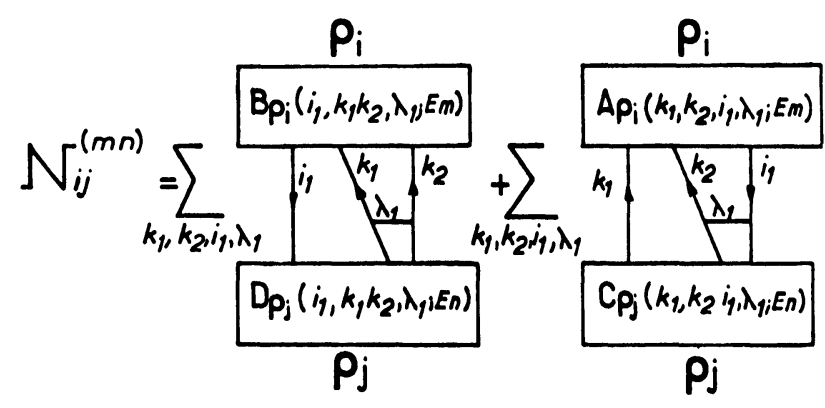

Fig. 8. - The metric $N$ as defined by equation (13).

Once the wavefunctions are known one can proceed to calculate any transition operator through the corresponding graphic representation [8]. Some deficiencies of a first order NFT treatment appear in these general equations. Let us assume that we choose as basic states only states of type $|\sigma\rangle$ which are more than enough to span the physical space. From equations $(4 a)$ and $(4 b)$ it is obvious that the resulting dynamical matrix $W^{(1)}(E)$ depends only on $\omega_{n}(0 \lambda), A_{\sigma}^{(1)}$ and $\Lambda_{n}(0 \lambda)$ - quantities which are independent of the particle-particle interaction. Thus, in that case, the results of a first order NFT treatment are insensitive to the particle-particle interaction. In a similar way if one considers only basic states of type $|\pi\rangle$, it is obvious from figure 3 or equation $(6 a)$ that $W_{\pi \pi^{\prime}}^{(1)}(E)=0$ and hence a first order treatment is, in that case, a zero order treatment, the energies of which are independent of the particle-hole interaction. These conclusions do not hold in a full order treatment. Since the shell-model results depend on both particle-particle and particle-hole interactions, one can invert the argument and say that a first order NFT treatment finds it an advantage to mix basic states of type $|\sigma\rangle$ and $|\pi\rangle$.

3. Comparison with shell-model. - In this section we will apply the equations derived above to illustrate and to check the theory as well as to gain insight into the interplay among the various NFT degrees of freedom. For this purpose we consider a two level model (and, thus, only one kind of nucleons, either neutrons or protons). The single-particle level is labelled by the letter $k$ while $i$ is the single-hole level. These two levels are separated by an energy $\varepsilon$ with $\varepsilon_{k}=0$, such that the bosons energies are given by

$$
\begin{aligned}
& \omega_{n}(0 \lambda)=\varepsilon+\left\langle k i^{-1} ; \lambda|V| k i^{-1} ; \lambda\right\rangle \\
& \omega_{n}(2 \lambda)=\langle k k ; \lambda|V| k k ; \lambda\rangle
\end{aligned}
$$

where $n=1$. 
The pairing boson carries an angular momentum $\lambda=0,2, \ldots, 2 k-1$, so that the maximum total angular momentum is $I_{\max }=2 k+i-1$. For the particle-hole boson, instead,

$$
i=|k-i|,|k-i|+1, \ldots, k+i .
$$

Therefore there is a state with $I=2 k+i$ which must be spurious. We will first analyse this state.

The only possible basic state is, then,

$$
\left[c_{k}^{\dagger} \Gamma_{1}^{\dagger}(0 k+i)\right]_{I}|0\rangle
$$

and the only intermediate state is $\left|\left[k\left(k i^{-1}\right)_{k+i}\right]_{I}\right\rangle$. In this very simple case one readily finds from equation (A.4)

$$
\begin{array}{r}
K_{a a}=(2 k+2 i+1)\left\{\begin{array}{lll}
k & i & k+i \\
k & I & k+i
\end{array}\right\} V_{1} \eta^{-1}= \\
=-V_{1} \eta^{-1}
\end{array}
$$

and equation $(6 a)$ gives

$$
A^{(1)}=-V_{1} \eta^{-1}
$$

where

$$
\eta=E-\varepsilon
$$

$$
\begin{aligned}
V_{1}=\left\langle k i^{-1} ; k+i|V| k i^{-1}\right. & ; k+i\rangle= \\
& =\Lambda_{1}\left(k i^{-1}, 0 k+i\right)
\end{aligned}
$$

then equation (5) reads

$$
A=-V_{1} /\left(V_{1}+\eta\right)
$$

while the propagator $C$ is in the same fashion found to be

$$
C=-A
$$

and the matrix element (4) is

$$
W=\omega_{1}(0 k+i)+V_{1} A
$$

which implies, from equation (11), $E_{n}=\varepsilon$. Thus, the only solution has an energy which coincides with the intermediate state energy. The norm of this state can be evaluated making use of equations (13) and (19) to get

$$
\langle\Psi \mid \Psi\rangle=1+\lim _{\eta \rightarrow 0} A C=0 .
$$

As already proved in the three identical particle case [8], the spurious state has, here also, an energy equal to an intermediate state energy and a norm equal to zero.

The complexity of the theory increases enormously with the dimension of the basis. This can be seen analysing just the next possible state, namely

$$
I=2 k+i-1 \text {. }
$$

In this case there are three basic states,

$$
\begin{aligned}
& |1\rangle \equiv\left|\sigma_{1}\right\rangle=\left[c_{k}^{\dagger} \Gamma_{1}^{\dagger}\left(0 \lambda_{1}\right)\right]_{I}|0\rangle \\
& |2\rangle \equiv\left|\sigma_{2}\right\rangle=\left[c_{k}^{\dagger} \Gamma_{1}^{\dagger}\left(0 \lambda_{2}\right)\right]_{I}|0\rangle \\
& |3\rangle \equiv|\pi\rangle=\left[b_{i}^{\dagger} \Gamma_{1}^{\dagger}\left(2 \lambda_{3}\right)\right]_{I}|0\rangle
\end{aligned}
$$

where $\lambda_{1}=k+i, \lambda_{2}=k+i-1$ and $\lambda_{3}=2 k-1$. There are also three intermediate states, they are

$$
\begin{aligned}
& |a\rangle=\left|\left[k\left(k i^{-1}\right) \lambda_{1}\right]_{I}\right\rangle \\
& |b\rangle=\left|\left[k\left(k i^{-1}\right) \lambda_{2}\right]_{I}\right\rangle
\end{aligned}
$$

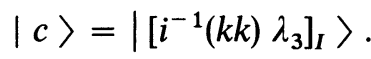

In the following we make use of the symmetric matrix $L$ such that

$$
\begin{gathered}
L_{11}=\hat{\lambda}_{1}^{2}\left\{\begin{array}{lll}
k & i & \lambda_{1} \\
k & I & \lambda_{1}
\end{array}\right\}=k / \lambda_{1} \\
L_{12}=-\hat{\lambda}_{1} \hat{\lambda}_{2}\left\{\begin{array}{lll}
k & i & \lambda_{1} \\
k & I & \lambda_{2}
\end{array}\right\}=-(i(2 k+i))^{1 / 2} / \lambda_{1} \\
L_{22}=\hat{\lambda}_{2}^{2}\left\{\begin{array}{lll}
k & i & \lambda_{2} \\
k & I & \lambda_{2}
\end{array}\right\}=-L_{11}
\end{gathered}
$$

$L_{13}=\hat{\lambda}_{1} \hat{\lambda}_{3}\left\{\begin{array}{ccc}k & k & \lambda_{3} \\ i & I & \lambda_{1}\end{array}\right\}=\left((2 k+i) / 2 \lambda_{1}\right)^{1 / 2}$

$L_{23}=\hat{\lambda}_{2} \hat{\lambda}_{3}\left\{\begin{array}{ccc}k & k & \lambda_{3} \\ i & I & \lambda_{2}\end{array}\right\}=-\left(i / 2 \lambda_{1}\right)^{1 / 2}$

$L_{33}=0$

(notice that $\operatorname{Tr} L=0$, det $L=1$ ). With the notation $L_{i j}^{-1}=\left(L^{-1}\right)_{i j}$ and after some algebra one finds

$$
\begin{aligned}
& W_{11}(E)=V_{1}+\varepsilon+V_{1}^{2}\left(L_{11} \eta^{2}-\eta\left(V_{0} L_{33}^{-1}+2 V L_{22}^{-1}\right)+2 V_{0} V\right) / d \\
& W_{12}(E)=V_{1} V_{0} \eta\left(L_{12} \eta+2 V L_{12}^{-1}\right) / d \\
& W_{13}(E)=\sqrt{2} V_{1} V \eta\left(L_{13} \eta+V_{0} L_{13}^{-1}\right) / d \\
& W_{22}(E)=V_{0}+\varepsilon+V_{0}^{2}\left(L_{22} \eta^{2}-\eta\left(V_{1} L_{33}^{-1}+2 V L_{11}^{-1}\right)+2 V_{1} V\right) / d \\
& W_{23}(E)=\sqrt{2} V_{0} V \eta\left(L_{23} \eta+V_{1} L_{23}^{-1}\right) / d \\
& W_{33}(E)=V+\varepsilon+2 V^{2}\left(L_{33} \eta^{2}-\eta\left(V_{1} L_{22}^{-1}+V_{0} L_{11}^{-1}\right)+V_{1} V_{0}\right) / d
\end{aligned}
$$


where $\eta$ and $V_{1}$ are as before (Eq. (18)) and

$$
\begin{gathered}
V_{0}=\left\langle k i^{-1} ; \lambda_{2}|V| k i^{-1} ; \lambda_{2}\right\rangle ; \quad V=\left\langle k k ; \lambda_{3}|V| k k ; \lambda_{3}\right\rangle ; \\
d=\eta^{3}-\eta^{2}\left(V_{1} L_{11}+V_{0} L_{22}+2 V L_{33}\right)+\eta\left(V_{1} V_{0} L_{33}^{-1}+2 V_{1} V L_{22}^{-1}+2 V_{0} V L_{11}^{-1}\right)-2 V_{1} V_{0} V .
\end{gathered}
$$

The diagonalization of the matrix $W$ can now be performed to find

$$
\eta^{2}\left(-\eta+\left(1+L_{11}\right) V_{1}+\left(1+L_{22}\right) V_{0}+V\right)=0
$$

which gives the physical root, whose expression coincides with the shell-model one

$$
\eta=\left(1+L_{11}\right) V_{1}+\left(1+L_{22}\right) V_{0}+V
$$

and two spurious roots $\eta=0$. For these roots one can easily (as compared with the previous derivations) find the propagators $A, B, C$ and $D$ with which one evaluates the metric (14) to get

$$
N_{i \leqslant j}=L_{i j}^{-1} / f_{j},
$$

where $f_{j}=\sqrt{2}$ if $j=3$, otherwise $f_{j}=1$. Then equation (13) becomes

$$
\langle\Psi \mid \Psi\rangle=\xi^{\dagger}(1+N) \xi
$$

but equation (11) gives for $\eta \rightarrow 0$

$$
(1+N) \xi=0
$$

which means that the norm of the spurious states is also in this case zero.

One can then conclude that although the NFT uses a heavily overcomplete basis, it provides the exact shell-model results. The other obtained states are degenerate with energies equal to intermediate state energies and their norm is equal to zero. They cannot be reached neither decay and are therefore spurious.

It is somehow puzzling that the theory corrects itself without making explicit use of any transformation to a basis which has the right dimensions, as done in other methods [2, 4]. Moreover, although different diagrams in figure 1 describe different physical processes one can wonder whether all of them are to be included into the basis in order to get the correct results. In methods which do not consider the bosons as entities that are independent of their single-particle constituents, one does not need to consider all states equivalent to those in figure 1 to describe the exact shell-model states [13]. One still uses in those methods overcomplete basis (with non-orthogonal elements) due to physical reasons, but one obtains in all cases the exact result. In cases like the one analysed here, the basis equivalent to the diagram in figure $1 a$ or in figure $1 b$, does not matter which, would be enough to span the whole shell-model space.
It would seem natural that the same is true in NFT. We can here easily check whether that is the case by considering in the example above only the basis element $(22 c)$. Thus the matrix $W$ has the only element $W_{33}$ of equation (25). With that element one finds that the solutions of equation (11) are given by the roots of the equation

$$
\begin{aligned}
& \eta^{3}-\eta^{2}\left(V_{1} L_{11}+V_{0} L_{22}+V\right) \\
& -\eta\left(V_{1} V_{0}+V_{1} V+V_{0} V\right)-V_{1} V_{0} V=0
\end{aligned}
$$

none of which coincides with (27) or is zero.

In the same manner if one considers only states $\left|\sigma_{1}\right\rangle$ and $\left|\sigma_{2}\right\rangle(22 a)$ and $(22 b)$, the solutions of equation (11) are roots of

$$
\eta f_{5}(\eta)=0
$$

where $f_{5}(\eta)$ is a fifth degree polynomial in $\eta$ whose expression is unnecessary. One spurious root still remains at intermediate energy $\eta=0$ while roots of $f_{5}(\eta)=0$ represent the mixing of both the physical state and other spurious solutions.

One then finds that all possible NFT basis elements must be included in order to get the exact result, whether or not the equivalent single-particle representation of the basis is already overcomplete within a small subset of the total set of diagrams.

4. Influence of the boson collectivity and of the truncation of the space. - The above example is simple enough to be carried out analytically and sufficient to

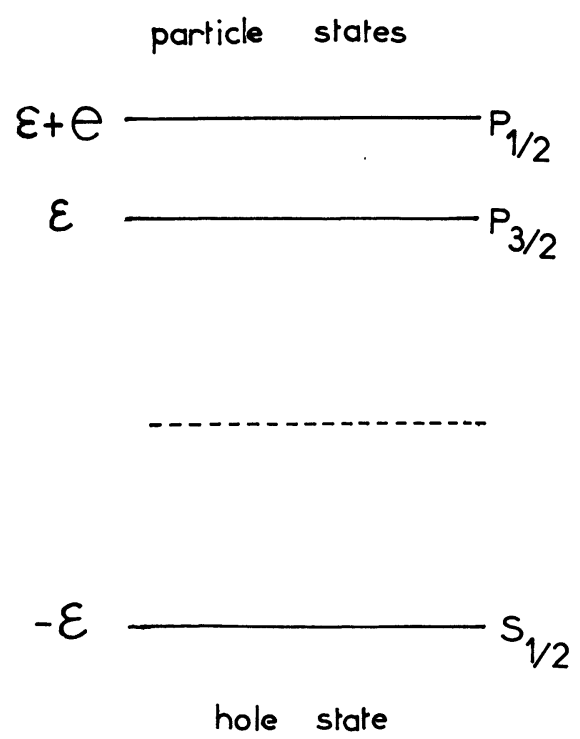

Fig. 9. - Single particle orbits and energies for the model of section 4. 
prove that the shell-model results are obtained only if all basic states are included and if the perturbation series is summed up to all orders. This conclusion is very important conceptually since the shell-model method is much easier than a proper (full order + all basic states) NFT treatment. In practice the Nuclear Field Theory is always used as an approximation of the shell-model. If the collectivity of the phonons is important one hopes that few basic states (partial treatment) are sufficient to give a good description of the lowest excited states. This statement cannot be studied with the above model since the corresponding phonons have only one component and thus never present collectivity. In order to remedy this drawback we present now another example, not solvable analytically, but easy to handle numerically.

Let us consider a single hole $s_{1 / 2}$ shell with energy $-\varepsilon$ and two single particle $\mathrm{p}_{3 / 2}$ and $\mathrm{p}_{1 / 2}$ shells with energies $\varepsilon$ and $\varepsilon+e$ respectively as shown in figure 9 .

Concerning the pairing bosons one has two $2^{+}$, two $0^{+}$states and one $1^{+}$state. Collectivity can occur for the $2^{+}$and $0^{+}$states. Concerning the surface bosons one has one $0^{-}$, one $2^{-}$states and two $1^{-}$ states allowing some collectivity again. It is easy to count three $1 / 2^{+}$, three $3 / 2^{+}$and two $5 / 2^{+} 2$-particle1 -hole states. In the following we focus our attention only to the $1 / 2^{+}$states, for which the $2^{+}$pairing bosons do not play any role.

Just to simplify the equations we suppose matrix elements of the form

$$
\begin{aligned}
\left\langle\mathrm{p}_{3 / 2} \mathrm{p}_{1 / 2} ; 1^{+}|V| \mathrm{p}_{3 / 2} \mathrm{p}_{1 / 2} ; 1^{+}\right\rangle=\left\langle\mathrm{p}_{3 / 2} \mathrm{p}_{3 / 2} ; 0^{+}|V| \mathrm{p}_{3 / 2} \mathrm{p}_{3 / 2} ; 0^{+}\right\rangle= \\
=\left\langle\mathrm{p}_{1 / 2} \mathrm{p}_{1 / 2} ; 0^{+}|V| \mathrm{p}_{1 / 2} \mathrm{p}_{1 / 2} ; 0^{+}\right\rangle=V_{0} ;\left\langle\mathrm{p}_{3 / 2} \mathrm{p}_{3 / 2} ; 0^{+}|V| \mathrm{p}_{1 / 2} \mathrm{p}_{1 / 2} ; 0^{+}\right\rangle=e \sqrt{a^{2}-1}
\end{aligned}
$$

for the particle-particle system $(a \geqslant 1)$ and

$$
\begin{aligned}
\left\langle\mathrm{p}_{1 / 2} \mathrm{~s}_{1 / 2}^{-1} ; 0^{-}|V| \mathrm{p}_{1 / 2} \mathrm{~s}_{1 / 2}^{-1} ; 0^{-}\right\rangle & =\left\langle\mathrm{p}_{3 / 2} \mathrm{~s}_{1 / 2}^{-1} ; 2^{-}|V| \mathrm{p}_{3 / 2} \mathrm{~s}_{1 / 2}^{-1} ; 2^{-}\right\rangle= \\
& =\left\langle\mathrm{p}_{3 / 2} \mathrm{~s}_{1 / 2}^{-1} ; 1^{-}|V| \mathrm{p}_{3 / 2} \mathrm{~s}_{1 / 2}^{-1} ; 1^{-}\right\rangle=\left\langle\mathrm{p}_{1 / 2} \mathrm{~s}_{1 / 2}^{-1} ; 1^{-}|V| \mathrm{p}_{1 / 2} \mathrm{~s}_{1 / 2}^{-1} ; 1^{-}\right\rangle \\
& =V_{1} ;\left\langle\mathrm{p}_{3 / 2} \mathrm{~s}_{1 / 2}^{-1} ; 1^{-}|V| \mathrm{p}_{1 / 2} \mathrm{~s}_{1 / 2}^{-1} ; 1^{-}\right\rangle=\frac{e}{2} \sqrt{b^{2}-1}
\end{aligned}
$$

for the particle-hole system $(b \geqslant 1)$. In addition to $\varepsilon$ and $e, V_{0}, V_{1}, a$ and $b$ are the only parameters of the problem (in fact one can express each energy in unit of $\varepsilon$ for instance). Let us proceed further and determine the pairing $\omega_{r}(2 \lambda)$ and surface $\omega_{r}(0 \lambda)$ bosons through the TDA equations.

$$
\begin{gathered}
\left(\omega_{r}(2 \lambda)-\varepsilon_{m}-\varepsilon_{n}\right) X_{m n}(r, 2 \lambda)=\sum_{p \leqslant q}\langle p q ; \lambda|V| m n ; \lambda\rangle X_{p q}(r, 2 \lambda) \\
\left(\omega_{r}(0 \lambda)-\varepsilon_{m}+\varepsilon\right) X_{m \mathrm{~s}_{1 / 2}}(r, 0 \lambda)=\sum_{n}\left\langle n \mathrm{~s}_{1 / 2}^{-1} ; \lambda|V| m \mathrm{~s}_{1 / 2}^{-1} ; \lambda\right\rangle X_{n \mathrm{~s}_{1 / 2}}(r, 0 \lambda) .
\end{gathered}
$$

One finds for the pairing bosons :

$$
\begin{gathered}
\omega\left(2,1^{+}\right)=2 \varepsilon+e+V_{0} ; X_{\mathrm{p}_{3 / 2} \mathrm{p}_{1 / 2}}\left(2,1^{+}\right)=1 ; \Lambda\left(\mathrm{p}_{3 / 2} \mathrm{p}_{1 / 2} ; 2,1^{+}\right)=V_{0} \\
\omega_{1}\left(2,0_{1}^{+}\right)=2 \varepsilon+e+V_{0}-e a ; \quad X_{\mathrm{p}_{3 / 2} \mathrm{p}_{3 / 2}}\left(2,0_{1}^{+}\right)=\sqrt{\frac{a+1}{2 a}} ; \quad X_{\mathrm{p}_{1 / 2} \mathrm{p}_{1 / 2}}\left(2,0_{1}^{+}\right)=-\sqrt{\frac{a-1}{2 a}} \\
\Lambda\left(\mathrm{p}_{3 / 2} \mathrm{p}_{3 / 2} ; 2,0_{1}^{+}\right)=\left[e(1-a)+V_{0}\right] \sqrt{\frac{a+1}{2 a}} ; \Lambda\left(\mathrm{p}_{1 / 2} \mathrm{p}_{1 / 2} ; 2,0_{1}^{+}\right)=\left[e(1+a)-V_{0}\right] \sqrt{\frac{a-1}{2 a}} \\
\omega_{2}\left(2,0_{2}^{+}\right)=2 \varepsilon+e+V_{0}+e a ; \quad X_{\mathrm{p}_{3 / 2} \mathrm{p}_{3 / 2}}\left(2,0_{2}^{+}\right)=\sqrt{\frac{a-1}{2 a}} ; \quad X_{\mathrm{p}_{1 / 2} \mathrm{p}_{1 / 2}}\left(2,0_{2}^{+}\right)=\sqrt{\frac{a+1}{2 a}} \\
\Lambda\left(\mathrm{p}_{3 / 2} \mathrm{p}_{3 / 2} ; 2,0_{2}^{+}\right)=\left[e(1+a)+V_{0}\right] \sqrt{\frac{a-1}{2 a}} ; \Lambda\left(\mathrm{p}_{1 / 2} \mathrm{p}_{1 / 2} ; 2,0_{2}^{+}\right)=\left[e(a-1)+V_{0}\right] \sqrt{\frac{a+1}{2 a}}
\end{gathered}
$$

and for the surface bosons

$$
\begin{gathered}
\omega\left(0,0^{-}\right)=2 \varepsilon+e+V_{1} ; \quad X_{\mathrm{p}_{1 / 2} \mathrm{~s}_{1 / 2}}\left(0,0^{-}\right)=1 ; \quad \Lambda\left(\mathrm{p}_{1 / 2} \mathrm{~s}_{1 / 2} ; 0,0^{-}\right)=V_{1} \\
\omega\left(0,2^{-}\right)=2 \varepsilon+e+V_{1} ; \quad X_{\mathrm{p}_{3 / 2} \mathrm{~s}_{1 / 2}}\left(0,2^{-}\right)=1 ; \quad \Lambda\left(\mathrm{p}_{3 / 2} \mathrm{~s}_{1 / 2} ; 0,2^{-}\right)=V_{1} \\
\omega_{1}\left(0,1_{1}^{-}\right)=2 \varepsilon+\frac{e}{2}+V_{1}-\frac{e}{2} b ; \quad X_{\mathrm{p}_{3 / 2} \mathrm{~s}_{1 / 2}}\left(0,1_{1}^{-}\right)=\sqrt{\frac{b+1}{2 b} ; \quad X_{\mathrm{p}_{1 / 2} \mathrm{~s}_{1 / 2}}\left(0,1_{1}^{-}\right)=-\sqrt{\frac{b-1}{2 b}}}
\end{gathered}
$$




$$
\begin{gathered}
\Lambda\left(\mathrm{p}_{3 / 2} \mathrm{~s}_{1 / 2} ; 0,1_{1}^{-}\right)=\left[\frac{e}{2}(1-b)+V_{1}\right] \sqrt{\frac{b+1}{2 b}} ; \Lambda\left(\mathrm{p}_{1 / 2} \mathrm{~s}_{1 / 2} ; 0,1_{1}^{-}\right)=\left[\frac{e}{2}(1+b)+V_{1}\right] \sqrt{\frac{b-1}{2 b}} \\
\omega_{2}\left(0,1_{2}^{-}\right)=2 \varepsilon+\frac{e}{2}+V_{1}+\frac{e b}{2} ; \quad X_{\mathrm{p}_{3 / 2} \mathrm{~s}_{1 / 2}}\left(0,1_{2}^{-}\right)=\sqrt{\frac{b-1}{2 b}} ; \quad X_{\mathrm{p}_{1 / 2} \mathrm{~s}_{1 / 2}}\left(0,1_{2}^{-}\right)=\sqrt{\frac{b+1}{2 b}} \\
\Lambda\left(\mathrm{p}_{3 / 2} \mathrm{~s}_{1 / 2} ; 0,1_{2}^{-}\right)=\left[\frac{e}{2}(1+b)+V_{1}\right] \sqrt{\frac{b-1}{2 b}} ; \Lambda\left(\mathrm{p}_{1 / 2} \mathrm{~s}_{1 / 2} ; 0,1_{2}^{-}\right)=\left[\frac{e}{2}(b-1)+V_{1}\right] \sqrt{\frac{b+1}{2 b}} .
\end{gathered}
$$

From equations (33) and (34) it appears that the parameter $a$ (and $b$ ) is the only one that influences the collectivity of the pairing (and surface) boson. For $a=1$ (and $b=1$ ) the bosons are pure in structure while for $a \rightarrow \infty$ (and $b \rightarrow \infty$ ) they present a maximum of collectivity.

Lastly let us consider the three $1 / 2^{+}$two-particle-one-hole states. After some Racah algebra it can be shown that their shell-model energies $E_{1}, E_{2}, E_{3}$ are the eigenvalues of the dynamical matrix

$$
\left(\begin{array}{ccc}
3 \varepsilon+V_{0}+2 V_{1} & e \sqrt{a^{2}-1} & \frac{e}{4} \sqrt{3\left(b^{2}-1\right)} \\
e \sqrt{a^{2}-1} & 3 \varepsilon+2 e+V_{0}+2 V_{1} & -\frac{e}{4} \sqrt{6\left(b^{2}-1\right)} \\
\frac{e}{4} \sqrt{3\left(b^{2}-1\right)} & -\frac{e}{4} \sqrt{6\left(b^{2}-1\right)} & 3 \varepsilon+e+V_{0}+2 V_{1}
\end{array}\right)
$$

In the proper NFT treatment there are nine basic states namely

$$
\begin{gathered}
\left|\mathrm{p}_{3 / 2} ; 2^{-}\right\rangle,\left|\mathrm{p}_{3 / 2} ; 1_{1}^{-}\right\rangle,\left|\mathrm{p}_{3 / 2} ; 1_{2}^{-}\right\rangle,\left|\mathrm{p}_{1 / 2} ; 0^{-}\right\rangle,\left|\mathrm{p}_{1 / 2} ; 1_{1}^{-}\right\rangle,\left|\mathrm{p}_{1 / 2} ; 1_{2}^{-}\right\rangle, \\
\left|\mathrm{s}_{1 / 2}^{-1} ; 0_{1}^{+}\right\rangle,\left|\mathrm{s}_{1 / 2}^{-1} 0_{2}^{+}\right\rangle,\left|\mathrm{s}_{1 / 2}^{-1} ; 1^{+}\right\rangle
\end{gathered}
$$

showing the big overcompleteness of the basis and ten intermediate states namely :

$$
\begin{gathered}
\left|p_{3 / 2}\left(p_{3 / 2} s_{1 / 2}^{-1}\right) 2^{-}\right\rangle,\left|p_{3 / 2}\left(p_{3 / 2} s_{1 / 2}^{-1}\right) 1^{-}\right\rangle,\left|p_{3 / 2}\left(p_{1 / 2} s_{1 / 2}^{-1}\right) 1^{-}\right\rangle, \\
\left|p_{1 / 2}\left(p_{1 / 2} s_{1 / 2}^{-1}\right) 0^{-}\right\rangle, \quad\left|p_{1 / 2}\left(p_{3 / 2} s_{1 / 2}^{-1}\right) 1^{-}\right\rangle, \quad\left|p_{1 / 2}\left(p_{1 / 2} s_{1 / 2}^{-1}\right) 1^{-}\right\rangle, \quad\left|s_{1 / 2}^{-1}\left(p_{3 / 2} p_{3 / 2}\right) 0^{+}\right\rangle, \\
\left|s_{1 / 2}^{-1}\left(p_{1 / 2} p_{1 / 2}\right) 0^{+}\right\rangle,\left|s_{1 / 2}^{-1}\left(p_{3 / 2} p_{1 / 2}\right) 1^{+}\right\rangle,\left|s_{1 / 2}^{-1}\left(p_{1 / 2} p_{3 / 2}\right) 1^{+}\right\rangle .
\end{gathered}
$$

Let us now describe the procedure followed in this example. We choose $l$ basic states among the nine possible ones $(l \leqslant 9)$. Then we take an input energy $E_{\mathrm{i}}$ and compute $l$ first order propagators

$$
F_{\rho_{j}}^{(1)}\left(E_{\mathrm{i}}\right)=\left(\begin{array}{l}
A_{\rho_{j}}^{(1)}\left(E_{\mathrm{i}}\right) \\
B_{\rho_{j}}^{(1)}\left(E_{\mathrm{i}}\right)
\end{array}\right) \quad(j=1, \ldots, l)
$$

using equations $(6 a-6 d)$.

The second step is the calculation of the $10 \times 10$ kernel $K\left(E_{\mathrm{i}}\right)$ (Eq. (A.4)) and the inversion of system $K\left(E_{\mathrm{i}}\right) F_{\rho_{j}}\left(E_{\mathrm{i}}\right)=F_{\rho_{j}}^{(1)}\left(E_{\mathrm{i}}\right)$ (Eq. (5)) to get the exact propagators $F_{\rho_{j}}\left(E_{\mathrm{i}}\right)$. In a first order-treatment where $F^{(1)}$ is used instead of $F$ this second step is omitted. We proceed further by computing the dynamical $l \times l$ matrix $W\left(E_{\mathrm{i}}\right)=W^{(0)}\left(E_{\mathrm{i}}\right)+F\left(E_{\mathrm{i}}\right) \Lambda$ (Eqs. $(4 a-$ $4 b)$ ) and lastly diagonalize it to obtain $l$ eigenvalues $E_{0}\left(E_{\mathrm{i}}\right)$. It is convenient to plot the multivalued function $\delta E\left(E_{\mathrm{i}}\right)=E_{0}\left(E_{\mathrm{i}}\right)-E_{\mathrm{i}}$ as a function of the input energies $E_{\mathrm{i}}$. A NFT solution $E_{\mathrm{p}}$ is obtained for

$$
\delta E\left(E_{\mathrm{p}}\right)=0 .
$$

In the following applications we fix once for all the values of parameters $\varepsilon, e, V_{0}$ and $V_{1}$ to be 5, 2,
$-1,-1.5$ respectively. In order to study the influence of the boson collectivity, four extreme cases are considered.

a) $a=4, b=5$ very collective $0^{+} \mathrm{p}-\mathrm{p}$ bosons and $1^{-}$p-h bosons;

b) $a=1.1, b=5$ collective $1^{-} \mathrm{p}-\mathrm{h}$ bosons, noncollective $0^{+} \mathrm{p}$-p bosons ;

c) $a=4, b=1.1$ collective $0^{+}$p-p bosons, noncollective $1^{-} \mathrm{p}$-h bosons;

d) $a=1.1, b=1.1$ non-collective $0^{+} \mathrm{p}-\mathrm{p}$ bosons and $1^{-} \mathrm{p}-\mathrm{h}$ bosons.

Concerning the truncation of the basis, six different cases are studied

case $\mathrm{A}=$ the 9 possible basic states are included ; case $\mathrm{B}=$ all 6 basic states of type $|\sigma\rangle$ (Eq. $(3 a))$; 
case $\mathrm{C}=$ all 3 basic states of type $|\pi\rangle$ (Eq. $(3 b))$; case $\mathrm{D}=$ only $\left|\sigma_{1}\right\rangle=\left|\mathrm{p}_{3 / 2}, 1_{1}^{-}\right\rangle$ case $\mathrm{E}=$ only $\left|\pi_{1}\right\rangle=\left|\mathrm{s}_{1 / 2}^{-1}, 0_{1}^{+}\right\rangle$ case $\mathrm{F}=\left|\sigma_{1}\right\rangle+\left|\pi_{1}\right\rangle$ taken into account .

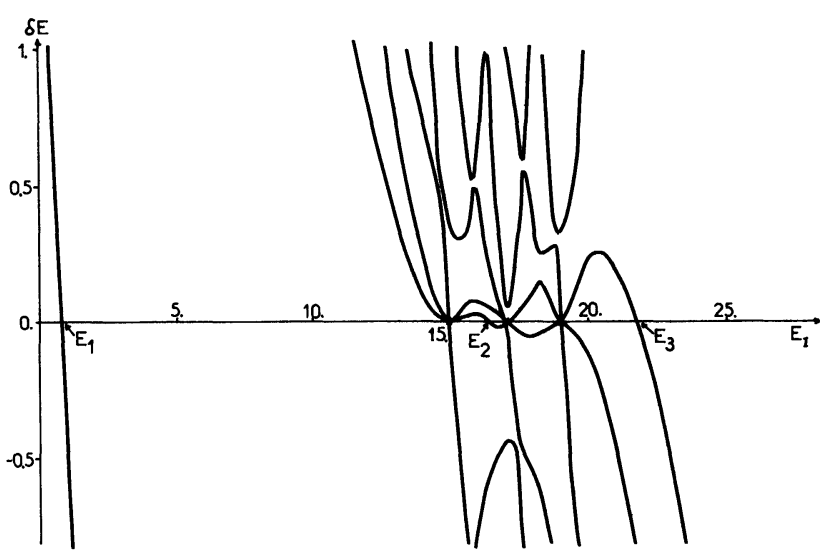

(a)

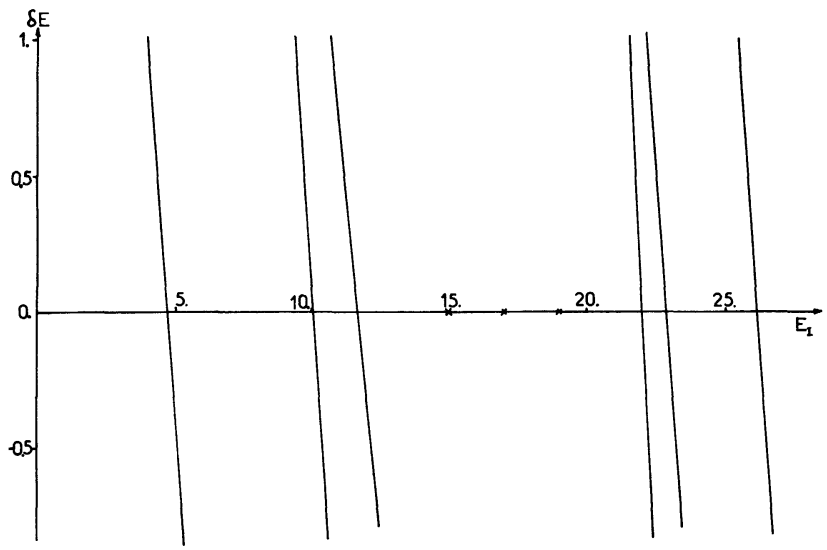

(b)

Fig. 10. - Full order (a) and first order $(b)$ NFT treatment for $1 / 2^{+}$ 2-particle-1-hole states studied in section 4 . The parameters are $\varepsilon=5, e=2, V_{0}=-1, V_{1}=-1.5, a=4, b=5$ and all basic states are included. The function $\delta E$ is defined in the text.

Figure $10 a$ shows the function $\delta E\left(E_{\mathrm{i}}\right)$ for the proper NFT treatment (case A) with $a=4, b=5$. The spurious solutions are concentrated at intermediate energies $3 \varepsilon=15,3 \varepsilon+e=17$ and $3 \varepsilon+2 e=19$ and therefore easy to remove. The remaining solutions $E_{1}, E_{2}, E_{3}$ coincide with the eigenvalues of (35) and therefore give the shell-model results. The conclusion of the previous section is emphasized again in this more elaborated model. In case of truncation (cases B and C for example) the situation is less clear : although some spurious states remain at the right energies, some of them are mixed with the physical ones. Anyhow the lowest physical state is always well isolated from other solutions. The first order treatment corresponding to figure $10 a$ is illustrated in figure $10 b$. One sees that the spurious states are no more easily disentangled from the physical ones. Moreover the lowest solution $E_{1}$ is shifted by some 4 energy units to the right; this is the net effect of the neglected diagrams.
Let us now analyse the effects of the boson collectivity and the truncation of the basis on the lowest physical state $E_{1}$. They are reported on table I where the upper row corresponds to a full order treatment while the lower row deals with a first order treatment only. As already pointed out, the first row of case A gives the shell-model results and hence " the exact solution ". In any case the values coming from a full order treatment are closer to the exact results than the corresponding first order ones. The neglected diagrams always correct the results of the 1st order in the right direction; the correction may be weak in case of no collectivity or very important in case of strong collectivity. We note however that in this peculiar model the effective degeneracy $\Omega_{\text {eff }}$ is very small and hence the higher order diagrams play an important role. In a more realistic situation the discrepancy between a full order and a first order NFT treatment would be greatly reduced.

Table I. - Values of the lowest physical energy for different collectivity parameter $a$ and $b$ (column a-d) and different truncation of the space (line A-F). The meaning of these various cases is explained in the text. The upper row is the value resulting from a full order NFT treatment while the lower row corresponds to a first order treatment.

\begin{tabular}{|c|c|c|c|c|}
\hline & $\mathrm{a}$ & $\mathrm{b}$ & $\mathrm{c}$ & $\mathrm{d}$ \\
\hline $\mathrm{A}$ & 0.855 & 5.240 & 4.946 & 10.686 \\
& 4.77 & 7.73 & 7.39 & 12.11 \\
\hline \multirow{2}{*}{ B } & 3.24 & 5.43 & 9.49 & 11.04 \\
& 7.88 & 7.88 & 12.52 & 12.52 \\
\hline \multirow{2}{*}{ C } & 3.61 & 9.66 & 5.34 & 11.68 \\
& 8.00 & 13.80 & 8.00 & 13.80 \\
\hline D & 5.13 & 7.20 & 14.32 & 11.67 \\
& 10.50 & 10.50 & 14.05 & 14.05 \\
\hline \multirow{2}{*}{ E } & 3.65 & 9.78 & 5.37 & 11.73 \\
& 8.00 & 13.80 & 8.00 & 13.80 \\
\hline \multirow{2}{*}{ F } & 2.35 & 7.03 & 5.26 & 11.28 \\
& 6.16 & 10.22 & 7.81 & 12.97 \\
\hline
\end{tabular}

When the particle-particle $0_{1}^{+}$boson is collective while the particle-hole $1_{1}^{-}$boson is not (column c) one sees that the use of only the lowest basic state of type $|\pi\rangle$ (line E) gives a very good approximation of the true eigenstate. Indeed adding two more $|\pi\rangle$ states (line C) or one more $|\sigma\rangle$ state (line F) have practically no effect on the result. On the other hand it is clear that basic states of type $|\sigma\rangle$ (lines D and B) are not suited for a good description of this state. 
When the particle-hole $1_{1}^{-}$boson is collective while the particle-particle $0_{1}^{+}$boson is not (column b) the reverse is true. The lowest $|\sigma\rangle$ state plays the most important role while type $|\pi\rangle$ states have only minor effects.

The case reported in column a where both particleparticle and particle-hole bosons are collective is especially instructive. The lowest $|\pi\rangle$ state (line E) is not sufficient to allow good description of $E_{1}$ and adding two more $|\pi\rangle$ states (line $C$ ) changes practically nothing. In a similar way the lowest $|\sigma\rangle$ state (line D) fails to reproduce $E_{1}$; adding five more $|\sigma\rangle$ states (line B) improves a bit the situation but not enough. If one considers $\left|\sigma_{1}\right\rangle$ and $\left|\pi_{1}\right\rangle$ as basic states (line $F$ ) the agreement is satisfactory. This result illustrates the empirical rule for truncating a space built in terms of a weak coupling basis (Refs. [4] and [8]) : to describe an " exact » eigenstate at energy $E$ it is necessary to include as basic states those whose zero order energies (here $\omega+\varepsilon$ ) are close to $E$. In the present case, this energy criterion implies a mixing of two different « types » of basic states.

5. Discussion and conclusions. - We showed in this paper how to sum-up the whole diagrammatic series of the nuclear field theory treatment for a two-particleone-hole system. This case is specially interesting from a physical point of view because one can test on equal footing the particle-particle as well as the particlehole interactions. From a more technical point of view this study is also interesting because several types of basic states (see Fig. 1) enter the formalism and make the NFT basis heavily overcomplete. The Brillouin-Wigner perturbation expansion was used in order to minimize the number of diagrams to be calculated. As compared to the first order NFT treatment, the full order treatment needs one additional step-namely the computation of the exact propagators $A$ and $B$ (see Eqs. (5)). This results in solving a set of linear systems, an operation which may be time consuming (if a large number of basic states are taken into account) but which presents no technical difficulties. The problem of solving the energy dependent Schrödinger equation (11) is much more delicate, but this drawback already exists in a first order treatment. Besides the method given to sum-up the whole diagrammatic series, other crucial points are emphasized in this paper. The spurious states coming from the violation of the Pauli principle in the NFT basis can be removed in a proper treatment. The spurious states have energies which coincide with intermediate state energies and, furthermore, their norms are equal to zero. They are null states. Once the spurious states have been removed, the remaining states, called physical states, present all the features of the shell-model states. Thus the conclusions of reference [8] are still valid in this more complicated case and ought to be true in any case if a proper NFT treatment is carried out. However the price to pay for a proper treatment is very high : not only the whole NFT series has to be calculated with the method developed in the present paper, but all possible NFT basic states ((1a) to (1d) for the 2-particle-1-hole system) have to be included (in peculiar basic states $(1 c)$ and $(1 d)$ which have always been neglected up to now are necessary). If one of these two conditions (or both as usually done) is not fulfilled the separation between spurious and physical states is not clear and some problems may arise. However a partial NFT treatment can be applied for those states where collective bosons play an important role; an energy criterion for selecting the basic states is then highly recommended. Even in that case a first order description may fail if shells with small degeneracy are influencial. In any case the elimination of spurious states is made easier in a full order treatment ; but then the numerical procedure gets much longer. This conclusion puts a severe limitation to the applicability of the Nuclear Field Theory.

Acknowledgments. - We are very indebted to R. Piepenbring for many valuable comments and criticisms of the manuscript.

Appendix. - From figure 5 one can write

$$
\begin{aligned}
A_{\rho}^{(m)}\left(k_{1}, k_{2} i_{1}, \lambda_{1} ; E\right)=\sum_{k_{3} i_{2} \lambda_{2}} K_{a a}\left(k_{1},\right. & \left.k_{2} i_{1}, \lambda_{1} ; k_{2}, k_{3} i_{2}, \lambda_{2} ; E\right) A_{\rho}^{(m-1)}\left(k_{2}, k_{3} i_{2}, \lambda_{2} ; E\right) \\
& +\sum_{k_{3} k_{4} \lambda_{2}} K_{a b}\left(k_{1}, k_{2} i_{1}, \lambda_{1} ; i_{1}, k_{3} k_{4}, \lambda_{2} ; E\right) B_{\rho}^{(m-1)}\left(i_{1}, k_{3} k_{4}, \lambda_{2} ; E\right)
\end{aligned}
$$

and a similar expression for the propagator $B_{\rho}$ (Fig. $5 b$ ).

Since the total propagators are defined as

$$
\begin{aligned}
& A_{\rho}=\sum_{m=1} A_{\rho}^{(m)} \\
& B_{\rho}=\sum_{m=1} B_{\rho}^{(m)}
\end{aligned}
$$


one obtain equation (5) where

$$
\begin{aligned}
& K_{a a}\left(k_{1}, k_{2} i_{1}, \lambda_{1} ; k_{2}, k_{3} i_{2}, \lambda_{2} ; E\right)= \\
& \quad=(-)^{\lambda_{2}-\lambda_{1}+k_{2}-k_{1}} \hat{\lambda}_{1} \hat{\lambda}_{2}\left\{\begin{array}{ccc}
k_{1} & i_{1} & \lambda_{2} \\
k_{2} & I & \lambda_{2}
\end{array}\right\}\left\langle k_{3} i_{2}^{-1} ; \lambda_{2}|V| k_{1} i_{1}^{-1} ; \lambda_{2}\right\rangle\left[E-\left(\varepsilon_{k_{1}}+\varepsilon_{k_{2}}-\varepsilon_{i_{1}}\right)\right]^{-1} \\
& K_{a b}\left(k_{1}, k_{2} i_{1}, \lambda_{1} ; i_{1}, k_{3} k_{4}, \lambda_{2} ; E\right)= \\
& \quad=\hat{\lambda}_{1} \hat{\lambda}_{2}\left\{\begin{array}{ccc}
k_{1} & k_{2} & \lambda_{2} \\
i_{1} & I & \lambda_{1}
\end{array}\right\} N\left(k_{3}, k_{4}\right) N\left(k_{1}, k_{2}\right)\left\langle k_{3} k_{4} ; \lambda_{2}|V| k_{2} k_{1} ; \lambda_{2}\right\rangle\left[E-\left(\varepsilon_{k_{1}}+\varepsilon_{k_{2}}-\varepsilon_{i_{1}}\right)\right]^{-1} \\
& K_{b a}\left(i_{1}, k_{1} k_{2}, \lambda_{1} ; k_{2}, k_{3} i_{2}, \lambda_{2} ; E\right)= \\
& \quad=\hat{\lambda}_{1} \hat{\lambda}_{2}\left\{\begin{array}{ccc}
i_{1} & k_{1} & \lambda_{2} \\
k_{2} & I & \lambda_{1}
\end{array}\right\}\left\langle k_{3} i_{2}^{-1} ; \lambda_{2}|V| k_{1} i_{1}^{-1} ; \lambda_{2}\right\rangle\left[E-\left(\varepsilon_{k_{1}}+\varepsilon_{k_{2}}-\varepsilon_{i_{1}}\right)\right]^{-1} .
\end{aligned}
$$

For the propagators $C_{\rho}$ and $D_{\rho}$ defined in figure 7 one proceeds as before to get equation (8) where

$$
\begin{aligned}
K_{c c}\left(k_{1}, k_{2} i_{1}, \lambda_{1} ; k_{3}, k_{1} i_{2}, \lambda_{2} ; E\right)= & (-)^{k_{1}-k_{3}+\lambda_{1}-\lambda_{2}} \hat{\lambda}_{1} \hat{\lambda}_{2}\left\{\begin{array}{ccc}
k_{1} & i_{2} & \lambda_{2} \\
k_{3} & I & \lambda_{1}
\end{array}\right\} \times \\
& \times\left\langle k_{2} i_{1}^{-1} ; \lambda_{1}|V| k_{3} i_{2}^{-1} ; \lambda_{1}\right\rangle\left[E-\left(\varepsilon_{k_{1}}+\varepsilon_{k_{2}}-\varepsilon_{i_{1}}\right)\right]^{-1} \\
K_{c d}\left(k_{1} ; k_{2} i_{1}, \lambda_{1} ; i_{2}, k_{3} k_{1}, \lambda_{2} ; E\right) & =\hat{\lambda}_{1} \hat{\lambda}_{2}\left\{\begin{array}{ccc}
k_{1} & k_{3} & \lambda_{2} \\
i_{2} & I & \lambda_{1}
\end{array}\right\} \times \\
& \times\left\langle k_{2} i_{1}^{-1} ; \lambda_{1}|V| k_{3} i_{2}^{-1} ; \lambda_{1}\right\rangle\left[E-\left(\varepsilon_{k_{1}}+\varepsilon_{k_{2}}-\varepsilon_{i_{1}}\right)\right]^{-1} \\
K_{d c}\left(i_{1}, k_{1} k_{2}, \lambda_{1} ; k_{3}, k_{4} i_{1}, \lambda_{2} ; E\right)= & \hat{\lambda}_{1} \hat{\lambda}_{2}\left\{\begin{array}{ccc}
i_{1} & k_{4} & \lambda_{2} \\
k_{3} & I & \lambda_{1}
\end{array}\right\} \times \\
& \times\left[E-\left(\varepsilon_{k_{1}}+\varepsilon_{k_{2}}-\varepsilon_{i_{1}}\right)\right]^{-1} N\left(k_{1} k_{2}\right) N\left(k_{3} k_{4}\right)\left\langle k_{1} k_{2} ; \lambda_{1}|V| k_{4} k_{3} ; \lambda_{1}\right\rangle .
\end{aligned}
$$

The propagators $C_{\rho}^{(1)}$ and $D_{\rho}^{(1)}$ can also be evaluated as done for $A_{\rho}$ and $B_{\rho}$ to obtain

$$
\begin{gathered}
C_{\sigma}^{(1)}\left(k_{1}, k_{2} i_{1}, \lambda_{1} ; E\right)=\delta\left(k_{1}, k\right) \delta\left(\lambda_{1}, \lambda\right) \Lambda_{n}\left(k_{2} i_{1}, 0 \lambda_{1}\right)\left[E-\left(\varepsilon_{k_{1}}+\varepsilon_{k_{2}}-\varepsilon_{i_{1}}\right)\right]^{-1} \\
D_{\sigma}^{(1)}\left(i_{1}, k_{1} k_{2}, \lambda_{1} ; E\right)=0 \quad C_{\pi}^{(1)}\left(k_{1}, k_{2} i_{1}, \lambda_{1} ; E\right)=0 \\
D_{\pi}^{(1)}\left(i_{1}, k_{1} k_{2}, \lambda_{1} ; E\right)=\delta\left(i_{1}, i\right) \delta\left(\lambda_{1}, \lambda\right) \Lambda_{n}\left(k_{1} k_{2}, 2 \lambda_{1}\right)\left[E-\left(\varepsilon_{k_{1}}+\varepsilon_{k_{2}}-\varepsilon_{i_{1}}\right)\right]^{-1} .
\end{gathered}
$$

\section{References}

[1] Beliaev, S. T. and Zelevinsky, V. G., Nucl. Phys. 39 (1962) 582 ;

Marumori, T., Yamamura, M. and Tokunaga, A., Prog. Theor. Phys. 31 (1964) 1.009.

Arima, A. and Iachello, F., Phys. Rev. C 16 (1977) 2085.

[2] Ko, C. M., Kuo, T. T. S. and McGrory, J. B., Phys. Rev. C 8 (1973) 2379.

[3] Ring, P. and Schuck, P., Z. Phys. 269 (1974) 323.

[4] Boisson, J. P., Silvestre-Brac, B. and Liotta, R. J., J. Physique Lett. 40 (1979) L-263; Nucl. Phys. A 330 (1979) 307.

[5] Bortignon, P. F., Broglia, R. A., Bes, D. R. and Liotta, R. J., Phys. Rep. 30 (1977) 305, and references therein.

Reinhard, H., Nucl. Phys. A 251 (1975) 317.

[6] Janouch, F. A. and Liotta, R. J., Phys. Lett. 82B (1979) 329 ; Nucl. Phys. A 334 (1980) 427.
[7] Broglia, R. A., Liotta, R., Nilsson, B. S., Dasso, C. H. and Winther, A., Phys. Rep. 29 (1977) 291.

[8] Liotta, R. J. and Silvestre-Brac, B. A., Nucl. Phys. A 309 (1978) 301.

[9] Bes, D. R., Dussel, G. G., Broglia, R. A., Liotta, R. J. and Motrelson, B. R., Phys. Lett. 52B (1974) 253.

[10] Bes, D. R. and Broglia, R. A., Phys. Rev. C 3 (1971) 2349.

[11] Намамoto, I., Phys. Rep. 10 (1974) 63.

[12] Bes, D. R., Broglia, R. A., Dussel, G. G., Liotta, R. J. and Perazzo, R. P. J., Nucl. Phys. A 260 (1976) 77.

[13] Silvestre-Brac, B., Thèse d'Etat, Université de Grenoble (1979) ;

Silvestre-Brac, B. and Boisson, J. P., to be published.

[14] Bertsch, G. F., Bortignon, P. F. and Broglia, R. A., Phys. Lett. 80B (1979) 161. 